\title{
Swift heavy ion irradiation effect on Cu-doped CdS nanocrystals embedded in PMMA
}

\author{
SHWETA AGRAWAL ${ }^{1, *}$, SUBODH SRIVASTAVA ${ }^{1}$, SUMIT KUMAR ${ }^{1}$, S S SHARMA ${ }^{1,2}$, \\ B TRIPATHI ${ }^{1}$, M SINGH ${ }^{1}$ and Y K VIJAY ${ }^{1}$ \\ ${ }^{1}$ Department of Physics, University of Rajasthan, Jaipur 302 004, India \\ ${ }^{2}$ Department of Physics, Govt. Women Engineering College, Ajmer 305 002, India
}

MS received 27 April 2009; revised 3 September 2009

\begin{abstract}
Semiconductor nanocrystals (NCs) have received much interest for their optical and electronic properties. When these NCs dispersed in polymer matrix, brightness of the light emission is enhanced due to their quantum dot size. The CdCuS NCs have been synthesized by chemical route method and then dispersed in PMMA matrix. These nanocomposite polymer films were irradiated by swift heavy ion (SHI) (100 MeV, $\mathrm{Si}^{+7}$ ions beam) at different fluences of $1 \times 10^{10}$ and $1 \times 10^{12}$ ions $/ \mathrm{cm}^{2}$ and then compared their structural and optical properties by XRD, atomic force microscopy, photoluminescence, and UV-Vis spectroscopy before and after irradiation. The XRD spectra showed a broad hump around $2 \theta \approx 11.83^{\circ}$ due to amorphous PMMA and other peaks corresponding to hexagonal structure of CdS nanocrystals in PMMA matrix. The photoluminescence spectra shows a broad peak at $530 \mathrm{~nm}$ corresponding to green emission due to $\mathrm{Cu}$ impurities in CdS. The UV-Vis measurement showed red shift in optical absorption and bandgap changed from 4.38-3.60 eV as the irradiation fluency increased with respect to pristine $\mathrm{CdCuS}$ nanocomposite polymer film.
\end{abstract}

Keywords. Nanocrystals (NCs); ultra violet-visible (UV-Vis); polymethylmethacrylate (PMMA); X-ray diffraction (XRD); photoluminescence (PL).

\section{Introduction}

Semiconductor CdS nanocrystals (NCs) have been widely synthesized and studied because they have unique properties and are suitable for photo reactivity and photo catalyst applications. The properties of $\mathrm{CdS}$ NCs are driven mainly by two factors, viz. large surface to volume ratio and a drastic change in electronic structure of material due to quantum mechanical effects with decreasing particle size (Prabhu and Khadar 2005). The ability to tune the bandgap of $\mathrm{CdS}$ by doping the impurity such as $\mathrm{Cu}, \mathrm{Ag}$ is another important factor which suits any specific application. Copper as an activator may occupy various sites in crystalline lattice of $\mathrm{CdS} \mathrm{NCs}$ as a result some additional emission centres create and depending on their concentration, either blue or green emission may occur (Bhargava et al 1994; Huang et al 1997; Xu et al 1998; Lee et al 2003; Mohanta et al 2003, 2006a; Kumar and Choudhary 2007). Que et al (1998) reported that $\mathrm{Cu}$ as an acceptor defect could give rise to localized level near to the valence band and luminescence arises from radiative recombination of electrons in conduction band with holes trapped at localized acceptor level above the valence

\footnotetext{
*Author for correspondence (agrw121@gmail.com)
}

band of CdS. Lui et al (2006) suggested that even small quantities of $\mathrm{Cu}$ incorporation result in variations in the PL and photoconductive properties. Doping of these impurity ions has been done by different techniques such as wet chemical process, co-evaporation, sol-gel method, thermal evaporation etc. These doped NCs have a wide range of applications in electroluminescence devices, light emitting displays and optical sensors (Mohanta et al 2004). Now a days the combination of semiconductor $\mathrm{NCs}$ with polymer matrix has received much attention due to their nonlinear optical properties. The advantage of such materials in polymers can be two-fold, first the tiny dots would enhance life of the polymer host and second the enhancement in light emission due to presence of quantum dots (Khanna and Singh 2007). The polymers prevent particles from agglomeration and provided a stable medium. A wide variety of materials modification in polymers has been studied by using ion irradiation technique. While traversing through matter, SHI loses its energy in two ways, nuclear energy losses $\left(S_{\mathrm{n}}\right)$ due to elastic collisions of projectile ions with atoms of the solids and electronic energy losses $\left(S_{\mathrm{e}}\right)$ due to inelastic collisions with the electrons of the solid medium. The electronic process, $S_{\mathrm{e}}$, involves $\mathrm{MeV}$ energies and use for introducing nanoscale defects (Columnar defects) as well as surface modifications. The interaction of high energy heavy 
ions with polymer, is associated with a considerable amount of linear electronic energy transfer (LET) by interactions (glancing as well as knock-on) with the orbital electrons of the target material, resulting in transient high local energy density deposition along the ion path. The knocked-out target electrons may transfer their energy into polymer matrix either by collision with other electrons or by electron phonon coupling. Consequently, huge electronic excitation and subsequent ionizations along the ion path/track triggers remarkable reorientation in the pristine structure leading to the ion beam modified zone. Therefore, the passage of SHI may give the information related to damage such as size, shape and structure of defects which changes the fluorescence property of semiconductor and this strongly depends on the mass of the incident ion, the irradiation energy and the fluence (Lesueur et al 1993; Avasthi 2000; Kanjilal 2001).

In this paper, we report the synthesis process of $\mathrm{Cu}$ doped $\mathrm{CdS}$ NCs and preparation of $\mathrm{CdCuS}$ doped nanocomposite polymer films by solution cast method. These nanocomposite polymer films were characterized by PL, UV-Vis spectroscopy, XRD and AFM techniques before and after SHI irradiation.

\section{Experimental}

\subsection{Materials}

Cadmium chloride $\left(\mathrm{CdCl}_{2}\right)$ and copper chloride $\left(\mathrm{CuCl}_{2}\right)$ were obtained from Sigma-Aldrich (99.9\%), dichloromethane was purchased from Merch Specialties Private Limited, Mumbai and polymethylmethacrylate (PMMA) granules were purchased from $\mathrm{M} / \mathrm{s}$ Gadra Chemicals, Bharuch. All chemicals were used as received.

\subsection{Synthesis of nanoparticles}

The NCs of $\mathrm{CdCuS}$ were synthesized by colloidal route method using Kibb's apparatus as shown in figure 1. Aqueous solution of cadmium chloride $\left(\mathrm{CdCl}_{2}\right)$ and copper chloride $\left(\mathrm{CuCl}_{2}\right)$ were prepared in deionized water. On reacting this mixture with $\mathrm{H}_{2} \mathrm{~S}$ gas for $1 \mathrm{~h}$, brownish solution was obtained. The precipitate was then washed with distilled water, filtered and dried in vacuum (Tripathi et al 2007).

\subsection{Preparation of polymer nanocomposite film}

The nanocomposite polymer films of $40 \mu \mathrm{m}( \pm 2 \mu \mathrm{m})$ thickness were prepared by dispersion of $(0.02 \mathrm{wt} . \%)$ $\mathrm{CdCuS} \mathrm{NCs}$ in $20 \mathrm{ml}$ of PMMA solution where dichloromethane was used as solvent (Awasthi et al 2006). This solution was stirred for $3 \mathrm{~h}$ on magnetic stirrer at room temperature and then purged in flat bottom petri dish floated over mercury for $24 \mathrm{~h}$ as shown in figure 2 . The nanocomposite film was pealed off from Petri dish and dried in vacuum for $2 \mathrm{~h}$ to completely remove the solvent.

\subsection{Swift heavy ion irradiation (SHI)}

As prepared nanocomposite polymer film was cut into the size of $1 \times 1 \mathrm{~cm}^{2}$ for SHI irradiation. These samples were irradiated by $\mathrm{Si}^{+7}$ ion beam (100 MeV energy) in the material science chamber under high vacuum $\left(\sim 10^{-6} \mathrm{mbar}\right)$, at $1 \times 10^{10}$ ions $/ \mathrm{cm}^{2}$ and $1 \times 10^{12}$ ions $/ \mathrm{cm}^{2}$, available from the $15 \mathrm{UD}$ tandem pelletron accelerator at IUAC, New Delhi, India (Mohanta et al 2006b).

\subsection{Characterization}

The XRD measurements of nanocomposite polymer films were performed with powder X-ray diffractometer. The optical absorption measurements were performed using a Hitachi-330 spectrophotometer. Photoluminescence were carried out using $\mathrm{He}-\mathrm{Cd}$ laser at a excitation wavelength, $325 \mathrm{~nm}$ and the surface topography of CdS films before and after irradiation was analysed with an atomic force microscopy (AFM), Digital Instruments Nanoscope III a. Measurements were taken in contact mode.

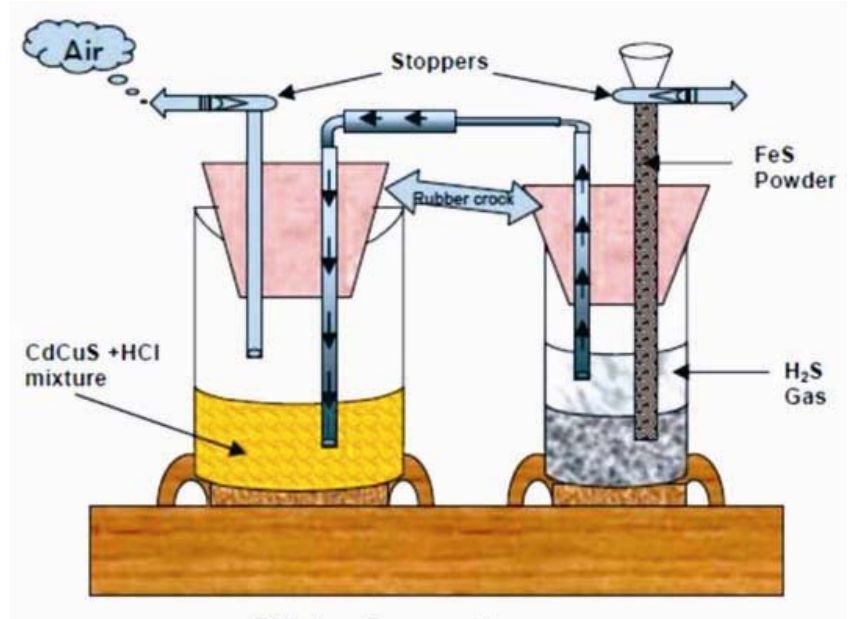

Kibbs Apparatus

Figure 1. The schematic diagram of Kibbs apparatus.

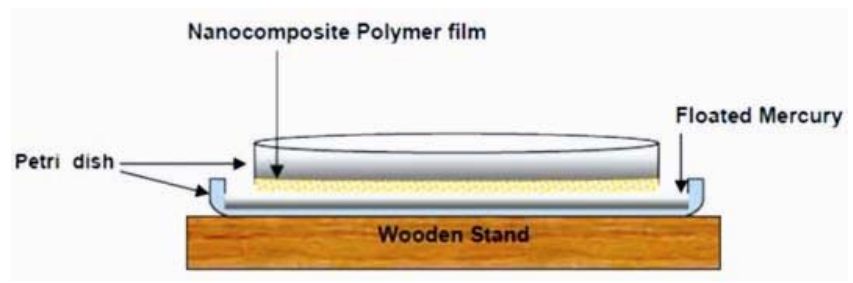

Figure 2. Schematic diagram of solution cast method for preparing the nanocomposite polymer film. 


\section{Results and discussion}

\subsection{X-ray diffraction}

The structural characteristics of nanocomposite polymer films before and after irradiation are shown in figure 3 . The XRD spectra shows a broad hump at $2 \theta \approx 11^{\circ}$ corresponding to amorphous nature of PMMA and other peaks appear at $2 \theta \approx 24.917^{\circ}, 26.627^{\circ}, 28 \cdot 326^{\circ}, 43.898^{\circ}, 48 \cdot 117^{\circ}$, these correspond to hexagonal structure of $\mathrm{CdS} \mathrm{NCs}$ as compared with standard JCPDS software. After irradiation reduction in peak intensity has been observed for all these samples (Liu et al 2003; Wang et al 2007).

\subsection{Optical absorption}

Figure 4 shows the absorbance vs wavelength spectra of $\mathrm{CdCuS}$ doped polymer film before and after irradiation. It is observed that the optical bandgap decreased after irradiation, possibly due to the combined effect of change in the grain size or agglomeration of quantum dots due to the interaction of heavy ion with nanocomposite polymer film. It has also been observed that the bandgap shifts from $4.38 \mathrm{eV}$ (figure $4 \mathrm{a}$ ) to $3.60 \mathrm{eV}$ (figure $4 \mathrm{c}$ ) as the fluence increases. The increase in grain size can be attributed to the fact that during irradiation the kinetic energy of the electrons ejected from the target atom is transferred to the compound lattice by electron-phonon interaction, which increases the local lattice temperature over the melting point of the material. We may suppose that the heat gets confined within the grain volume. This gives rise to non equilibrium state which, as a conse-

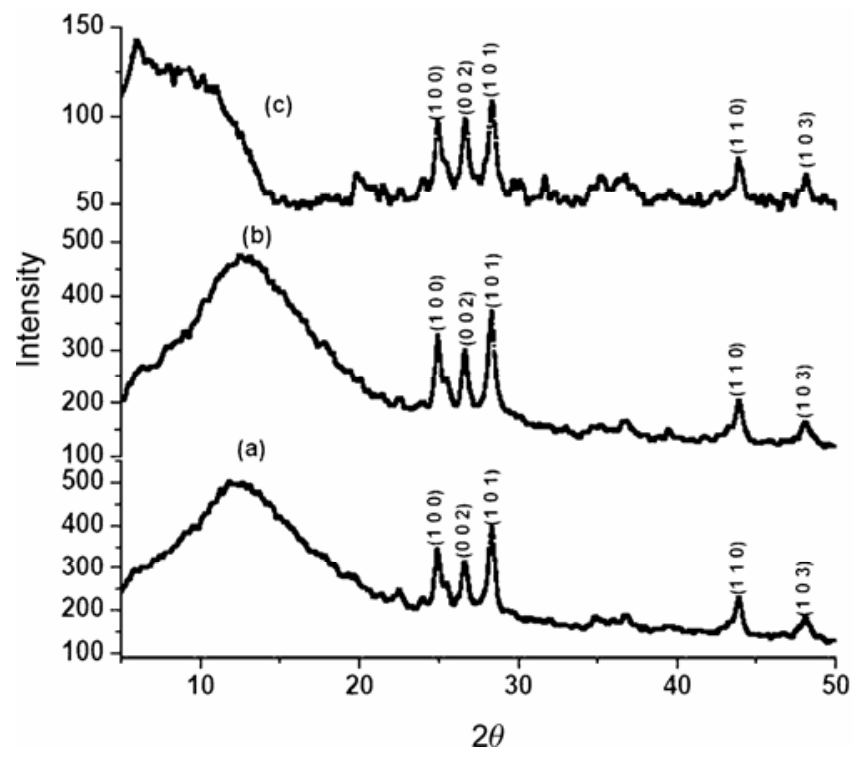

Figure 3. XRD pattern for (a) virgin $\mathrm{CdCuS}$ in PMMA, (b) $\mathrm{CdCuS}$ in PMMA irradiated at $1 \times 10^{10}$ ions $/ \mathrm{cm}^{2}$ and (c) $\mathrm{CdCuS}$ in PMMA irradiated at $1 \times 10^{12}$ ions $/ \mathrm{cm}^{2}$. quence, raises the volume of the grains resulting in grain agglomeration on irradiation.

\subsection{Photoluminescence}

Figure 5 shows PL spectra of pristine and irradiated nanocomposite polymer film. Figure 5(a) shows a luminescence peak, $\approx 530 \mathrm{~nm}$, corresponding to the energy of $2.33 \mathrm{eV}$, which is less than the bandgap energy. It is important to quote here that instead of well-defined peaks,

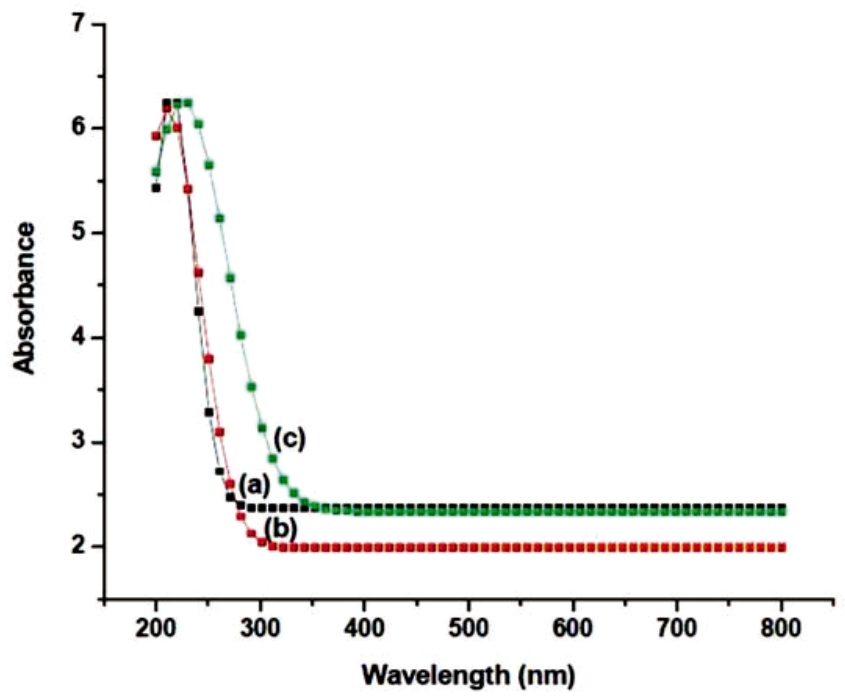

Figure 4. Optical absorption spectra of (a) virgin $\mathrm{CdCuS}$ in PMMA, (b) CdCuS in PMMA irradiated at $1 \times 10^{10}$ ions $/ \mathrm{cm}^{2}$ and (c) $\mathrm{CdCuS}$ in PMMA irradiated at $1 \times 10^{12}$ ions $/ \mathrm{cm}^{2}$.

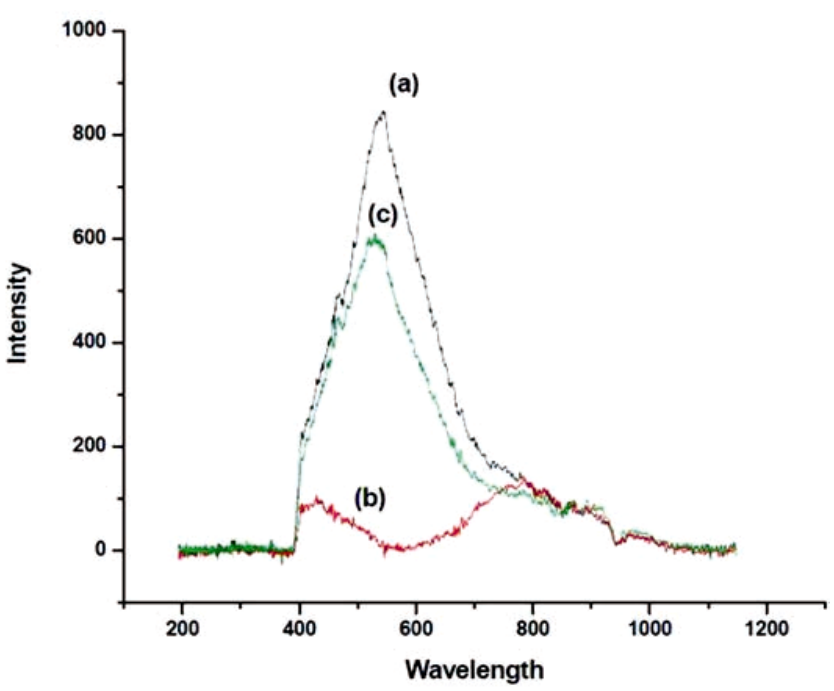

Figure 5. PL spectra of (a) virgin $\mathrm{CdCuS}$ in PMMA, (b) $\mathrm{CdCuS}$ in PMMA irradiated at $1 \times 10^{10}$ ions $/ \mathrm{cm}^{2}$ and (c) $\mathrm{CdCuS}$ in PMMA irradiated at $1 \times 10^{12}$ ions $/ \mathrm{cm}^{2}$. 


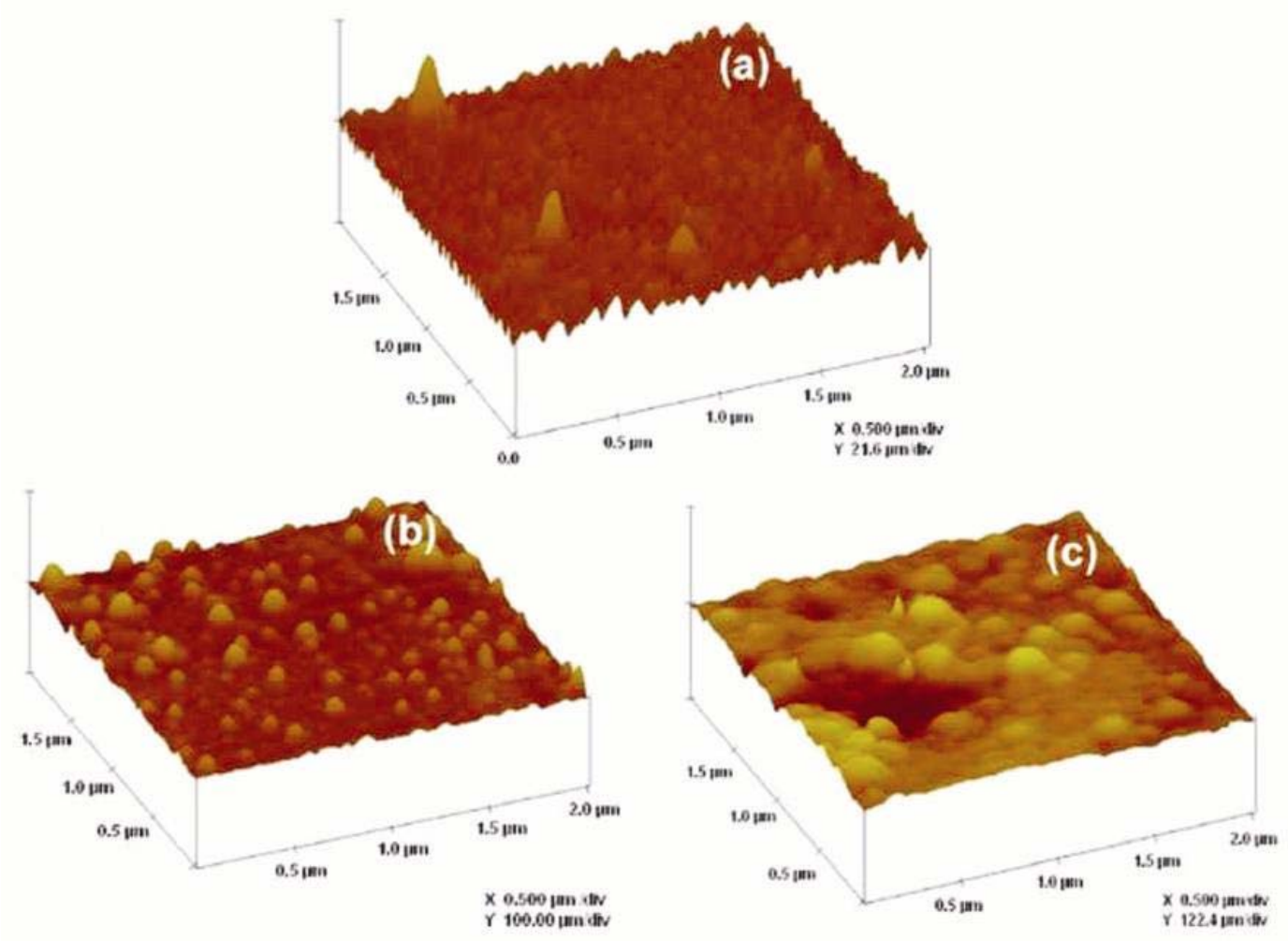

Figure 6. The atomic force micrographs of (a) virgin $\mathrm{CdCuS}$ in PMMA, (b) CdCuS in PMMA irradiated at $1 \times 10^{10}$ ions $/ \mathrm{cm}^{2}$ and (c) CdCuS in PMMA irradiated at $1 \times 10^{12}$ ions $/ \mathrm{cm}^{2}$.

the PL reveals broad band. Therefore, it may conclude that the band edge emission and deep trap emission are very much competitive due to heavy ion impact and finally merge into a single well-defined peak. As shown by our results, there is initial blue shift in the lower excitation wavelength which may attribute to enhanced non-radiative recombination due to additional deep levels at ion fluence of $1 \times 10^{10}$ ions $/ \mathrm{cm}^{2}$. At higher ion fluence of $1 \times 10^{12}$ ions $/ \mathrm{cm}^{2}$, the additional luminescence centres may form, which may trap electrons and holes and the fluorescence efficiency is increased, therefore, the emission peak shifts to longer wavelengths.

\subsection{Atomic force microscopy}

The AFM micrographs shown in figure 6 indicate a clear picture of the surface in nanoscale dimensions $(2 \times 2 \mu \mathrm{m}$ scan area), where the morphology predicts compact grain structure. From the micrograph shown in figure 6(a), it is evident that the surface of pristine nanocomposite polymer film is composed of dense grains. It has been observed that most of nanocrystals agglomerate and form an uneven cluster structure after irradiation as shown in figures 6(b) and (c). The effect of SHI irradiation on the surface roughness has also been investigated. The rootmean-square (RMS) surface roughness has been used to better understand the effect of irradiation. The measured
RMS values for $2 \times 2 \mu \mathrm{m}$ scan area is $1.313 \mathrm{~nm}$ for pristine films. While for irradiated films at $1 \times 10^{10}$ and $1 \times 10^{12}$ ions $/ \mathrm{cm}^{2}$ fluence RMS values are $3.906 \mathrm{~nm}$ and $6.27 \mathrm{~nm}$, respectively. This clearly shows that the surface roughness increases after irradiation.

\section{Conclusions}

The present study reveals that on SHI irradiation there are significant modifications in the structural and optical properties of nanocomposite polymer films. The stability of embedded $\mathrm{CdCuS}$ nanocrystals is more than that of its powder form. XRD confirms the hexagonal structure of $\mathrm{CdS}$ without any additional peak corresponding to copper. In photoluminescence the emission peak is observed in green region due to presence of $\mathrm{Cu}$ in $\mathrm{CdS}$. In optical absorption bandgap decreases with the fluence, this may be attributed to the formation of conjugated system of bonds due to bond cleavage and reconstruction. The effect of SHI irradiation on the surface roughness has also been investigated and it is observed that the surface roughness increases after irradiation.

\section{Acknowledgements}

The authors are thankful to IUAC, New Delhi, for providing ion irradiation facility to carry out this work and Dr 
F Singh and Pawan Kulhariya for experimental help during characterization.

\section{References}

\section{Avasthi D K 2000 Curr. Sci. 781297}

Awasthi Kamlendra, Kulshresta Vaibhav, Tripathi Balram, Acharya N K and Singh M 2006 Eur. Polym. J. 42883

Bhargava R N, Gallagher D, Hong X and Nurmikko A 1994 Phys. Rev. Lett. 72416

Huang Jinman, Yang Y I, Shanhua Xue, Bai Yang, Shiyong Liu and Jiacong Shen 1997 Appl. Phys. Lett. 70

Kanjilal D 2001 Curr. Sci. 801560

Khanna P K and Singh Narendra 2007 J. Lumin. 127474

Kumar Anil and Choudhary Vidhi 2007 J. Photochem. \& Photobiol. 189272

Lee Sangwook, Song Daegwon, Lee Jongwon, Kim Seontai, Park In Yong and Won Mi Sook 2003 Mater. Sci. \& Eng. B103 241

Lesueur D and Dunlop A 1993 Rad. Eff. \& Def. Solids 126163
Liu S H, Qian X F, Yin J, Ma X D, Yuan J Y and Zhu Z K 2003 J. Phys. \& Chem. Solids $\mathbf{6 4} 455$

Lui T Y et al 2006 Nanotechnology 175935

Mohanta D, Nath S S, Mishra N C and Choudhary A 2003 Bull. Mater. Sci. 26289

Mohanta D, Mishra N C and Choudhary A 2004 Mater. Letts 58 3694

Mohanta D, Ahmed G A, Choudhary A, Singh F and Awasthi D K 2006a J. Nanopart. Res. 8645

Mohanta D, Singh Fouran, Avasthi D K and Choudhary Amrjyoti 2006b Central Eur. J. Phys. 4187

Prabhu Rajeev R and Khadar M Abdul 2005 Pramana-J. Phys. 65801

Que Wenxiu, Zhou Y, Lam L, Chan Y C and Kam C H 1998 Appl. Phys. Letts 732727

Tripathi B, Singh F, Awasthi D K, Das D and Vijay Y K 2007 Physica B400 70

Wang Hongmei, Fang Pengfei, Chen Zhe and Wang Shaojie 2007 Appl. Surf. Sci. 2538495

Xu S J, Chua S J, Liu B, Gan L M, Chew C H and Xu G Q 1998 Appl. Phys. Lett. 73478 